\title{
Infrared Fine-Structure Line Diagnostics of Shrouded Active Galactic Nuclei
}

\section{N $93-26870$}

\author{
G. M. Voit (Caltech)
}

Far-infrared spectroscopy of celestial objects will improve dramatically in the coming decade, allowing astronomers to use fine-structure line emission to probe photoionized regions obscured in the optical band by thick clouds of dust. The ultraluminous far-IR galaxies revealed by IRAS, quasar-like in luminosity but smothered in molecular gas, probably conceal either immense starbursts or luminous active nuclei. In both scenarios, these objects ought to produce copious infrared fine-structure emission with several lines comparable to $\mathrm{H} \beta$ in luminosity. This paper shows how these lines, if detected, can be used to determine the electron densities and far-IR obscurations of shrouded photoionized regions and to constrain the shape and ionization parameter of the ionizing spectra. The presence of [Ne V] emission in particular will distinguish shrouded AGNs from shrouded starbursts. Since all active galaxies photoionize at least some surrounding material, these diagnostics can also be applied to active galaxies in general and will aid in studying how an active nucleus interacts with the interstellar medium of its host galaxy.

For more details, see the November 10, 1992 edition of the Astrophysical Journal.

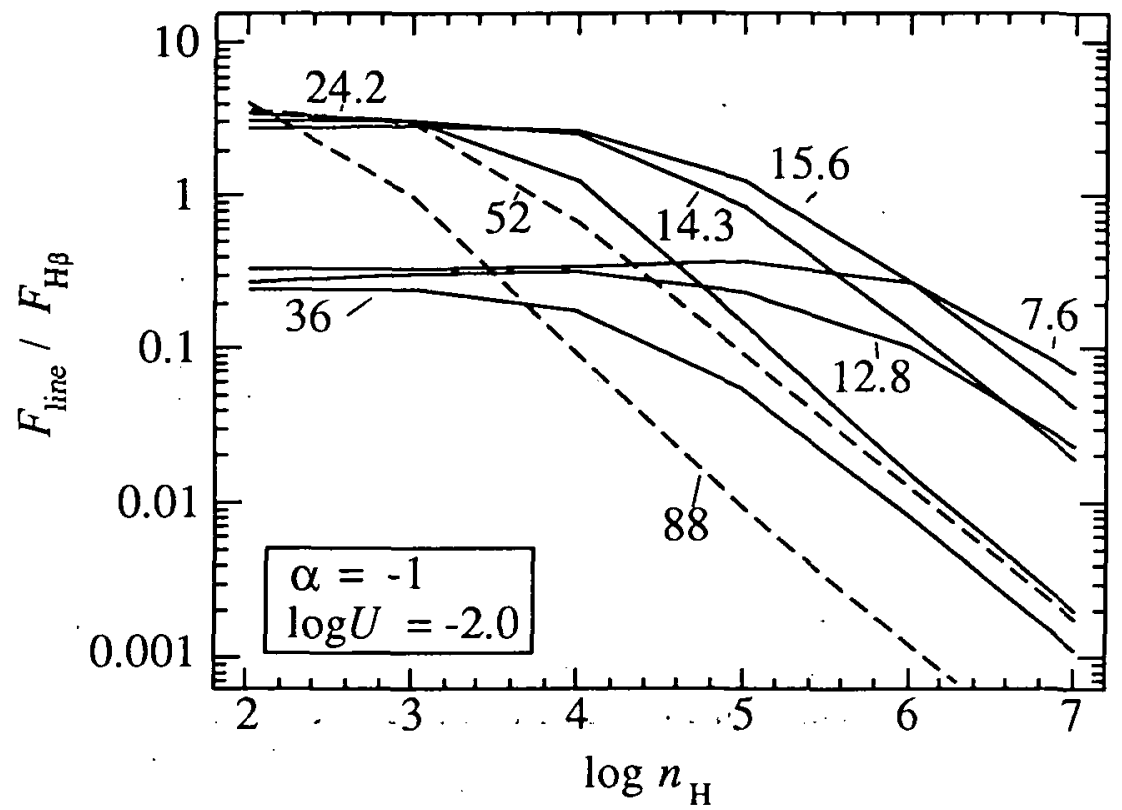

The strongest IR fine-structure lines from a photoionized region radiate as least as much power as the $\mathrm{H} \beta$ line, unless the electron density is very high. This figure illustrates the line fluxes, relative to $\mathrm{H} \beta$, for selected fine-structure transitions of oxygen and neon in an AGN-irradiated cloud of ionization parameter log $U=-2$. Labels on the curves give the wavelength of each transition in microns: [O III] 52 \& 88, [Ne II] 12.8, [Ne III] 15.6 \& 36.0, [Ne V] 14.3 \& 24.2, [Ne VI] 7.6: Note that the critical densities for quenching the neon lines are large $(\log n \sim 5-6)$. 


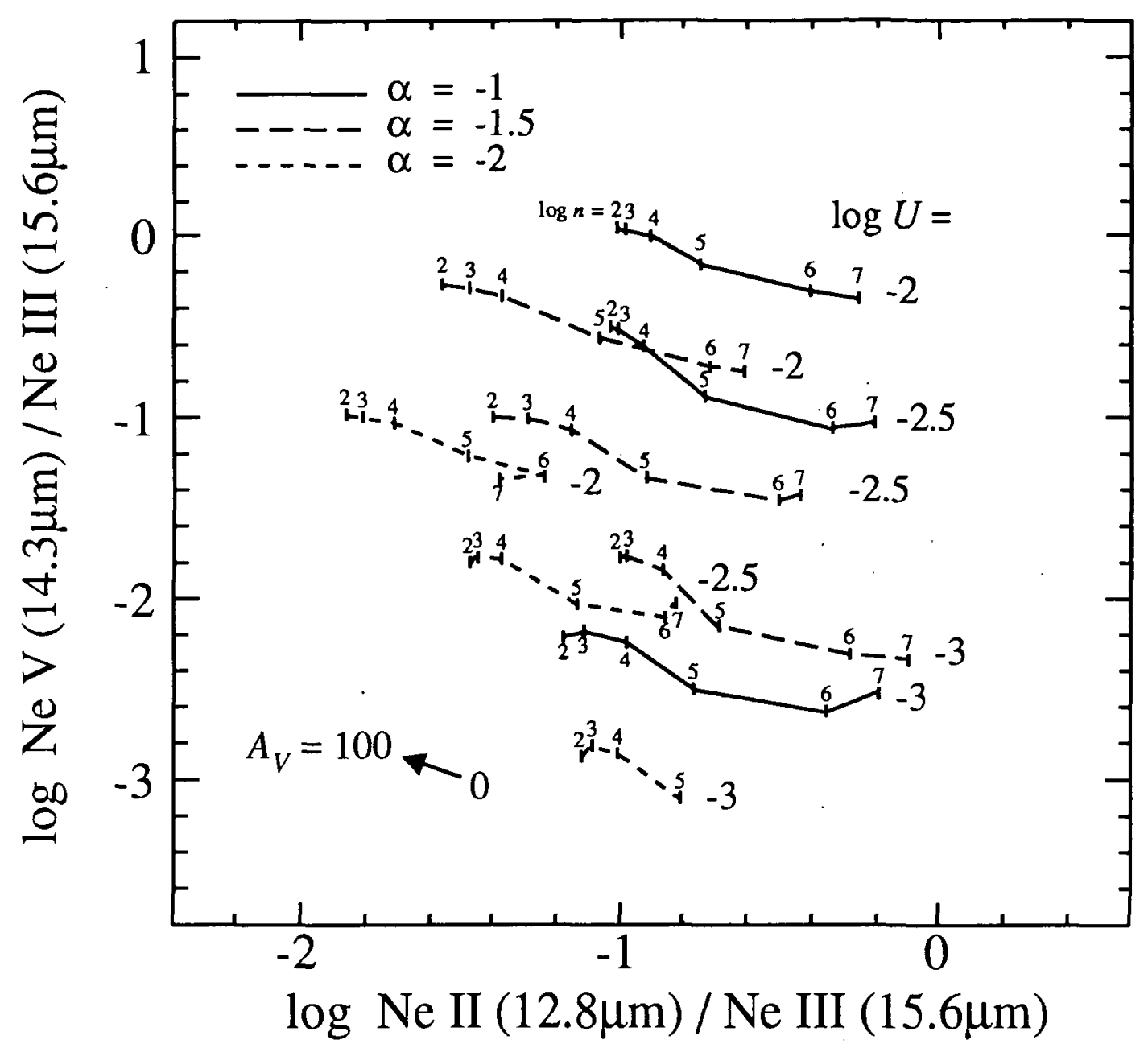

The [Ne II] $12.8 \mu \mathrm{m}$, [Ne III] $15.6 \mu \mathrm{m}$, and [Ne V] $14.3 \mu \mathrm{m}$ lines are all reddened by the about the same amount in the ISM and have critical densities that differ by an order of magnitude or less. Thus, they form an excellent ionization-diagnostic trio that probes a wide range of ionization parameters and photon energies. The neon-line plane above shows how these line ratios respond to different spectral shapes (power-laws with a ranging from -1 to -2 ) and ionization parameters ( $\log U$ ranging from -2 to -3 ) over a range of hydrogen densities ( $\log n$ ranging from 2 to 7 ). Reddening of $100 \mathrm{~V}$ magnitudes produces the shift indicated by the arrow. The $[\mathrm{Ne} \mathrm{V}] /[\mathrm{Ne}$ III] ratio is particularly sensitive to the ionization parameter, and the [Ne II]/[Ne III] ratio is somewhat sensitive to the hardness of the incident spectrum. Stellar $\mathrm{H}$ II regions lie far below the pictured portion of the plane, and shocks, with [Ne II]/[Ne III] >> 1 , lie far to the right. 